\title{
Integrability in the Theory of Schrödinger Operator and Harmonic Analysis
}

\author{
Oleg A. Chalykh and Alexander P. Veselov \\ Department of Mathematics and Mechanics, Moscow State University, 119899 Moscow, Russia
}

Received June 15, 1992

\begin{abstract}
The algebraic integrability for the Schrödinger equation in $\mathbb{R}^{n}$ and the role of the quantum Calogero-Sutherland problem and root systems in this context are discussed. For the special values of the parameters in the potential the explicit formula for the eigenfunction of the corresponding Sutherland operator is found. As an application the explicit formula for the zonal spherical functions on the symmetric spaces $S U_{2 n}^{*} / S p_{n}$ (type A II in Cartan notations) is presented.
\end{abstract}

\section{Introduction}

The discovery of the method of inverse scattering transformation in the sixties began a new era in the history of integrable systems. The stormy development of the theory of integrability influenced various domains of mathematics and mathematical physics. First of all it is concerned with the spectral theory. In particular, in 1974-76 there were discovered the beautiful results in the spectral theory of the Schrödinger operator

$$
L=-\frac{d^{2}}{d x^{2}}+u(x)
$$

with a periodic potential $u(x)$ (see [1] and references there). It turned out that such a spectral property of $L$ as finite-gapness is equivalent to the existence of commuting differential operator $A$ of odd order:

$$
[L, A]=0, \quad A=\frac{d^{2 n+1}}{d x^{2 n+1}}+\ldots,
$$

or the existence of the eigenfunction $\psi$, which is determined on the algebraic curve (see [1]).

This example demonstrates very well the phenomenon, which we would like to call as the integrability in the theory of the Schrödinger operator. In the present paper we continue the investigations of the multidimensional case begun in [2]. 
Recall that the quantum problem with the hamiltonian

$$
H=p^{2}+U(x), \quad p=i \frac{\partial}{\partial x}, \quad x \in \mathbb{R}^{n}
$$

and the corresponding Schrödinger equation

$$
L \psi=-\Delta \psi+U(x) \psi=E \psi
$$

is called integrable, if there exist $n$ commuting differential operators $L_{1}=L, L_{2} \ldots, L_{n}$ with algebraically independent highest symbols $P_{1}=\xi^{2}$, $P_{2}(\xi), \ldots, P_{n}(\xi)$.

Suppose we have one more operator $L_{0}$, commuting with $L_{i}(i=1, \ldots, n)$ and independent of them. More precisely, we will assume that the highest symbol $P_{0}(\xi)$ of $L_{0}$ takes the distinct values at the roots of the equations $P_{i}(\xi)=E_{i}(i=1, \ldots, n)$ for almost all $E_{i}$. In this case we will call Schrödinger equation (3) algebraically integrable.

Problem. To describe all algebraically integrable Schrödinger equations.

This problem even in a more general form was first investigated by I.M. Krichever, who proved in particular that the corresponding joint eigenfunction $\psi$ actually is determined on some algebraic variety [3]. But the first nonseparable examples of such commutative rings were found much later in [2]. We call the Schrödinger operator a separable if it commutes with an operator in a smaller number of variables. An example of such operators is given by

$$
L=-\Delta+u_{1}\left(x_{1}\right)+\cdots+u_{n}\left(x_{n}\right)
$$

where $u_{i}(x)$ are the finite-gap potentials for all $i=1, \ldots, n$.

In the paper [2] it was conjectured that all nonseparable algebraically integrable Schrödinger equations in dimension $n>1$ are nothing but multi-dimensional analogues of Lamé equations, ${ }^{1}$ connected with root systems of Lie algebra:

$$
L=-\Delta+\sum_{\varepsilon \in R_{+}} \ell_{\alpha}\left(\ell_{\alpha}+1\right)(\alpha, \alpha) \mathscr{P}((\alpha, x))
$$

and their degenerations. Here $R_{+}$is the set of positive roots of Lie algebra $G, \mathbb{R}^{n}$ is identified with its Cartan subalgebra $H \approx H^{*}, \mathscr{P}$ is the Weierstrass elliptic function, $\ell_{\alpha}$ are integers. In [2] $\ell_{\alpha}$ were assumed to be equal, but further investigations showed that at least in degenerate cases (see below) $\ell_{\alpha}$ should be allowed to depend on the length of $\alpha$.

We are still not able to investigate the elliptic case and want to present here some results concerning the degenerations of (4):

$$
L=-\Delta+\sum_{\alpha \in R_{+}} \ell_{\alpha}\left(\ell_{\alpha}+1\right)(\alpha, \alpha) f((\alpha, x)),
$$

\footnotetext{
1 This conjecture does not contradict the recent result of J. Feldman, H. Knörrer and E. Trubowitz [4], because all operators (4) are singular on the reals
} 
where $f=\frac{\omega^{2}}{\sinh ^{2} \omega z}, \frac{\omega^{2}}{\sin ^{2} \omega z}$ or $\frac{1}{z^{2}}$. The last case can be obtained as the limit of the first one when $\omega \rightarrow 0$. The first two cases come to each other after replacing $\omega \rightarrow i \omega$.

For the root system $A_{n-1}$ and $f=\frac{\omega}{\sin ^{2} \omega z}$ the corresponding operators (5) are the special cases of the Sutherland operator [5]

$$
L=-\Delta+\sum_{i<j} \frac{g}{\sin ^{2} \omega\left(x_{i}-x_{j}\right)}
$$

and for $f=\frac{1}{x^{2}}$ the Calogero operator $[6]$

$$
L=-\Delta+\sum_{i<j} \frac{g}{\left(x_{i}-x_{j}\right)^{2}} .
$$

The elliptic generalizations have appeared after Moser's investigation [7] of the corresponding classical systems. Olshanetsky and Perelomov were the first to propose the generalization of (6) and(7) using the root systems [8-10].

It is very interesting that the operator (6) for $g=-\frac{1}{2}$ has appeared already in 1964 in the paper [11] by Berezin et al. There is a remark in [11], which says that the radial part of the Laplace-Beltrami operator for the symmetric space of type AI is conjugated to such operator $L$, but it was left without further development: Only in 1978 Olshanetsky and Perelomov [12] proved the following general fact.

Let $X=G / K$ be any symmetric space (SS) of negative curvature, $R$ be the system of its roots $\alpha$ with the multiplicities $m_{\alpha}$ (see [13]). Let us introduce the function

$$
\xi(x)=\prod_{\alpha \in R_{+}}\left(\frac{\omega}{\sinh \omega(x, \alpha)}\right)^{m_{\alpha} / 2}
$$

and denote by $\hat{\xi}$ the operator of multiplication by $\xi, \hat{\xi}(f)=\xi f$.

Theorem [12]. The radial part $B$ of the Laplace-Beltrami operator on the space $X$ is conjugated to the operator

$$
\begin{gathered}
L=-\Delta+\sum_{\alpha \in R_{+}} g_{\alpha} \frac{\omega^{2}}{\sinh ^{2} \omega(\alpha, x)}-\rho^{2}, \\
g_{\alpha}=\frac{1}{4} m_{\alpha}\left(m_{\alpha}+2 m_{2 \alpha}-2\right)(\alpha, \alpha), \quad \rho=\frac{1}{2} \sum_{\alpha \in R_{+}} m_{\alpha} \alpha
\end{gathered}
$$

by means of the operator $\hat{\xi}$ :

$$
L=-\hat{\xi} \circ B \circ \hat{\xi}^{-1}
$$

These special values of $g_{\alpha}$ are sometimes called group values. The comparison of the formulas (5) and (8) together with the table of all irreducible SS (see [10, 13]) shows that the radial part of the Laplace-Beltrami operator is conjugated to the Schrödinger operator of the form (5) only when SS $X$ has a complex group of motion or has the type AII or EIV. ${ }^{2}$ For SS with the complex group $G$ the

\footnotetext{
${ }^{2}$ It is very interesting to notice that the SS of type AII and EIV has the quaternion and octonian groups of motion correspondently, so "integrable" SS coincide with the SS, connected with the algebras over $\mathbb{R}$ with division
} 
Laplace-Beltrami operator is conjugated to the trivial case $\ell_{\alpha}=0: L=\Delta$. This fact was used by Berezin [14] to find the spherical functions on such SS. For the type AII, i.e. for the SS $X=S U_{2 n}^{*} / S p_{n}$, corresponding Schrödinger operator has the form

$$
H=-\Delta+\sum_{i<j} \frac{4 \omega^{2}}{\sinh ^{2} \omega\left(x_{i}-x_{j}\right)} .
$$

We will show now that this operator is algebraically integrable in the above sense, and moreover we will find explicitly its eigenfunction, proving some conjectures in [2]. As a corollary we give the explicit formulas for the zonal spherical functions of these SS. For such functions there exists the general Harish-Chandra formula, representing them as integral over the compact group $K$ (see [13] and below). As a by-product we have an explicit expression for this integral over $K=S p_{n}$. For $n=3$ and 4 as well as for the SS $X=E_{6} / F_{4}$ of type EIV such formulas can be extracted from $[15,16]$.

This paper was written when one of the authors (A.V.) was a guest of the Forschungsinstitut für Mathematik (ETH, Zürich). He is very thankful to Prof. J. Moser for the hospitality, and to Profs. J. Feldman, H. Knörrer and E. Trubowitz for the stimulating discussions.

\section{Construction of Some Algebraically Integrable Schrödinger Equations and Their Solutions}

The following construction is a small modification of that proposed in [2].

Let $A$ be a finite set of vectors $\alpha \in \mathbb{R}^{n}$ (for the beginning noncollinear) with the prescribed multiplicities $m_{\alpha} \in \mathbb{N}$. Let the function $\psi(k, x), k, x \in \mathbb{R}^{n}$ have the following properties:

1) $\psi$ has the form

$$
\psi=P(k, x) \exp (k, x),
$$

where $P(k, x)$ is some polynomial on $k$ with the leading term

2) The relation

$$
A(k)=\prod_{\alpha \in A}(k, \alpha)^{m_{\alpha}} .
$$

$$
\psi(k+s \alpha, x) \equiv \psi(k-s \alpha, x)
$$

holds for all $\alpha \in A, s=1, \ldots, m_{\alpha}$ and for all $k$, belonging to the corresponding hyperplanes $(k, \alpha)=0$.

Remark. In the paper [2] the relation (12) is written in another, but equivalent, form

$$
\delta_{\alpha}\left((\alpha, k)^{-1} \delta_{\alpha}\right)^{s-1} \psi=0
$$

where

$$
\delta_{\alpha} \varphi(k, x):=\varphi(k+\alpha, x)-\varphi(k-\alpha, x) .
$$

Proposition 1. There exists no more than one function $\psi$ with these properties.

Proof. It is sufficient to prove that the leading term of the polynomial $P(k, x)$ for the function $\psi$ of the form (11) with the property (12) is divisible by $(k, \alpha)^{m_{\alpha}}$ for all $\alpha \in A$. But it easily follows from the formulas (13), which are equivalent to (12). 
The main question here is the existence of such functions $\psi$. In the paper [2] the authors conjectured that if $A$ is the system of positive roots of simple complex Lie algebra then the function $\psi$ with the prescribed properties does exist. The following result proves this conjecture.

Theorem 1. Let $A$ be the set of positive roots of simple complex Lie algebra $G$ with the multiplicities $m_{\alpha}$, depending only on the length of $\alpha$, then the function $\psi$ with the properties 1 and 2 exists and is unique.

Remark. The theorem is valid also for the nonreduced root system $B C_{n}$, if we replace the relations (12) for the roots $\alpha$ and $2 \alpha$ into

$$
\psi(k+s \alpha, x) \equiv \psi(k-s \alpha, x)
$$

for $(k, \alpha)=0$ and $s=1,2, \ldots, m_{\alpha}, m_{\alpha}+2, \ldots, m_{\alpha}+2 m_{2 \alpha}$.

The proof of this theorem uses the theory of generalized hypergeometric functions, developed by G. Heckman and E. Opdam in [17-21]. It is published in the separate paper [22].

Recently K. Styrkas proved the following theorem, which shows that under some assumptions the inverse statement also holds in a good agreement with the second conjecture of the paper [2].

Theorem (K. Styrkas [25]). If the set A consists of the mutually noncollinear vectors with unit multiplicities, then the function $\psi$ with the properties 1 and 2 exists only if $A \cup(-A)$ is the root system of some semisimple Lie algebra.

The following theorem explains the importance of such functions $\psi$ for the theory of commutative rings of partial differential operators (PDO).

Let us consider the ring $R_{A}$, consisting of the polynomials $f(k)$, satisfying the relations (12):

$$
f(k+s \alpha) \equiv f(k-s \alpha)(\bmod (k, \alpha))
$$

for all $\alpha \in A, s=1, \ldots, m_{\alpha}$.

Theorem 2. If the function $\psi$ with the properties (11), (12) does exist, then to every polynomial $f(k) \in R_{A}$ corresponds a PDO $L_{f}\left(x, \frac{\partial}{\partial x}\right)$, such that

$$
L_{f} \psi(k, x)=f(k) \psi(k, x) \text {. }
$$

All such operators form a commutative ring, isomorphic to $R_{A}$.

The proof can be found actually in [2], but we present it here to show that the construction of $L_{f}$ is quite effective.

Lemma. Any function of the form $\phi=Q(k, x) \exp (k, x)$ with a polynomial on $k Q$ which satisfies the relations (12), can be represented in the form

for some $\mathrm{PDO} L$.

$$
\phi=L\left(x, \frac{\partial}{\partial x}\right) \psi
$$


Proof of lemma. It is easy to deduce from (12) that the leading term of $Q$ has to be divisible by $A(k)=\prod_{\alpha \in A}(k, \alpha)^{m_{x}}$. Let $r(k, x)$ be the corresponding quotient. For the operator $L_{1}=r\left(\frac{\partial}{\partial x}, x\right)$ the function

$$
\phi_{1}=\phi-L_{1} \psi
$$

also satisfies the relations (12) and has a similar form

$$
\phi_{1}=Q_{1}(k, x) \exp (k, x),
$$

but the degree of the polynomial $Q_{1}$ on $k$ is less than $\operatorname{deg} Q$. Repeating this procedure we come to the statement of lemma.

To conclude Theorem 2 from this lemma it is sufficient to consider the function

$$
\phi=f(k) \psi(k, x) \text {. }
$$

If $f$ belongs to $R_{A}$, then this function satisfies the relation (12) and by the lemma there exists an operator $L_{f}$ such that

$$
\phi=L_{f}\left(x, \frac{\partial}{\partial x}\right) \psi=f(k) \psi
$$

The commutativity of such operators $L_{f}$ and $L_{g}$ follows from the fact that the equality $L \psi \equiv 0$ for the differential operator $L$ means that $L \equiv 0$.

Remark that $f(k)=-k^{2}$ belongs to $R_{A}$ for all $A$. The corresponding operator has the form

$$
L=-\Delta+\sum_{\alpha \in A} \frac{m_{\alpha}\left(m_{\alpha}+1\right)(\alpha, \alpha)}{\sinh ^{2}(\alpha, x)} .
$$

We should recall that it works only if the function $\psi$ with the properties (11), (12) does exist.

We know that it is the case if $A$ is the set $R_{+}$of the positive roots (see Theorem 1). Any polynomial invariant under the Weyl group $W$, generated by the reflections in the planes $(\alpha, k)=0$, belongs to $R_{A}$. According to Chevalley theorem the algebra $S^{W}$ of $W$-invariant polynomials is freely generated by $n$ polynomials $P_{1}=k^{2}, P_{2}, \ldots, P_{n}$.

It is very important for us that the ring $R_{A}$ is much bigger than $S^{W}$. Indeed, any polynomial $p$ from the ideal, generated by

$$
q(k)=\prod_{\alpha \in A}\left((k, \alpha)^{2}-(\alpha, \alpha)^{2}\right) \cdots\left((k, \alpha)^{2}-m_{\alpha}^{2}(\alpha, \alpha)^{2}\right),
$$

satisfies the relations (12) and therefore belongs to $R_{A}$, but in general is not invariant under the Weyl group. It means that the corresponding commutative ring of PDO is supercomplete in the sense of [2] and Schrödinger equation

$$
-\Delta \psi+\sum_{\alpha \in R_{+}} \frac{m_{\alpha}\left(m_{\alpha}+1\right)(\alpha, \alpha)}{\sinh ^{2}(\alpha, x)^{2}} \psi=E \psi
$$

is algebraically integrable. 
These equations for the classical root systems have the following form

$$
\begin{aligned}
& A_{n}:-\Delta \psi+\sum_{i<j} \frac{2 m(m+1)}{\sinh ^{2}\left(x_{i}-x_{j}\right)} \psi=E \psi ; \\
& B C_{n}:-\Delta \psi+\left(\sum_{i=1}^{n} \frac{4 p(p+1)}{\sinh ^{2} 2 x_{i}}+\sum_{i=1}^{n} \frac{q(q+2 p+1)}{\sinh ^{2} x_{i}}\right. \\
&\left.\quad+\sum_{i<j} \frac{2 r(r+1)}{\sinh ^{2}\left(x_{i}-x_{j}\right)}+\sum_{i<j} \frac{2 r(r+1)}{\sinh ^{2}\left(x_{i}+x_{j}\right)}\right) \psi=E \psi ; \\
& D_{n}:-\Delta \psi+\left(\sum_{i<j} \frac{2 m(m+1)}{\sinh ^{2}\left(x_{i}-x_{j}\right)}+\sum_{i<j} \frac{2 m(m+1)}{\sinh ^{2}\left(x_{i}+x_{j}\right)}\right) \psi=E \psi .
\end{aligned}
$$

To complete the investigations we should only find the explicit formulas for the functions $\psi$, what we are going to do now for the root systems $A_{n}$ and $m=1$. As it was shown before it is the most interesting case for the theory of SS. Such formulas were first conjectured in [2] (see p. 606), now we want to give the proof.

Remark. In the recent very interesting paper [21] G. Heckman proposed another approach to the similar problem, using the Dunkl differential-difference operator. It is much more general but seems to be less effective in our concrete case.

\section{Explicit Formulas for the Function $\psi$ for the Root System $A_{n-1}, m=1$}

Let us introduce the differential operators $Q_{N}^{i_{1}, \ldots, i_{k}}$, where $i_{1}, \ldots, i_{k}$ and $N$ are distinct natural numbers, by the following recurrent procedure [2]

$$
\begin{aligned}
Q_{N}= & 1, \quad Q_{N}^{i}=\partial_{i}-\partial_{N}-2 \omega \operatorname{coth} \omega\left(x_{i}-x_{N}\right) \\
Q_{N}^{i_{1}, \ldots, i_{k}}= & Q_{N}^{i_{1}, \ldots, i_{k-1}}{ }_{\circ}\left(\partial_{i_{k}}-\partial_{N}\right)-2 \omega \operatorname{coth} \omega\left(x_{i_{k}}-x_{N}\right) Q_{N}^{i_{1}, \ldots, i_{k-1}} \\
& +\sum_{s=1}^{k-1} 2 \omega^{2} \sinh ^{-2} \omega\left(x_{i_{s}}-x_{i_{k}}\right) Q_{N}^{i_{1}, \ldots, i_{s}, \ldots, i_{k-1}}
\end{aligned}
$$

where the symbol $\hat{i}$ means that this index should be omitted and $\partial_{i}=\partial / \partial x_{i}$. It is easy to check that the operators $D_{s}=Q_{s}^{1, \ldots, s-1}$ can be found also by replacing $k_{i} \rightarrow \partial_{i}-\partial_{s}$ in the following polynomial on $k$

$$
\begin{aligned}
& D_{s}(k, x) \\
& =\exp \left(-\sum_{i<s} 2 \operatorname{coth} \omega\left(x_{i}-x_{s}\right) \frac{\partial}{\partial k_{s}}+\sum_{i<j<s} 2 \omega^{2} \sinh ^{-2}\left(x_{i}-x_{j}\right) \frac{\partial^{2}}{\partial k_{i} \partial k_{j}}\right) \prod_{i<s} k_{i} .
\end{aligned}
$$

Theorem 3. The function

$$
\psi(k, x)=D_{n} \circ D_{n-1} \circ \cdots \circ D_{2} \exp (k, x)
$$

satisfies the relations (12) and therefore is the eigenfunction of the commutative ring of PDO, containing the Schrödinger operator (10). 
Proof. It is sufficient to prove that the operator

$$
D=D_{n} \circ D_{n-1} \circ \cdots \circ D_{2}
$$

is right-divisible by

$$
Q_{j}^{i}=\left(\partial_{i}-\partial_{j}\right)-2 \omega \operatorname{coth} \omega\left(x_{i}-x_{j}\right)=\operatorname{sh} \omega\left(x_{i}-x_{j}\right) \circ\left(\partial_{i}-\partial_{j}\right) \circ \mathrm{sh}^{-1} \omega\left(x_{i}-x_{j}\right) .
$$

Indeed

$$
\begin{aligned}
Q_{j}^{i} \exp (k, x) & =\operatorname{sh} \omega\left(x_{i}-x_{j}\right)\left(\partial_{i}-\partial_{j}\right) 2 \exp (k, x) \\
& =2\left(k_{i}-k_{j}\right) \operatorname{sh} \omega\left(x_{i}-x_{j}\right) \equiv 0 \bmod \left(k_{i}-k_{j}\right) .
\end{aligned}
$$

By the definition the operator $D$ is right-divisible by $Q_{2}^{1}$. We will prove now that the operator $D$ is anti-invariant under the permutation of coordinates

$$
\sigma_{*}(D)=(-1)^{\sigma} D, \quad \sigma \in S_{n} .
$$

Together with the previous remark it will imply the theorem.

Lemma 1. Let $u_{i j}=u\left(x_{i}-x_{j}\right), u(x)=2 \omega^{2} \sinh ^{-2} \omega x$. Then the following relation holds

$$
\left(\Delta-\sum_{i<j \leqq n} 2 u_{i j}\right) \circ Q_{n}^{1, \ldots, n-1}=Q_{n}^{1, \ldots, n-1} \circ\left(\Delta-\sum_{i<j \leqq n-1} 2 u_{i j}\right) \text {. }
$$

Proof of Lemma 1. Let us introduce the notations: $v(x)=2 \omega \operatorname{coth} \omega x$, $v_{i j}=v_{\hat{i}}\left(x_{i}-x_{j}\right), \partial_{i j}=\partial_{i}-\partial_{j}, \quad Q=Q_{n}^{1, \ldots, n-1}, \quad Q^{i}=Q=Q_{n}^{1, \ldots, \hat{i}, \ldots, n-1}, Q^{i j}=$ $Q_{n}^{1, \ldots, \hat{i}, \ldots, \hat{j}, \ldots, n-1}$, etc. Then the formulas (16) will have a form

$$
Q=Q^{i} \partial_{i n}-v_{i n} Q^{i}+\sum_{j \neq i} u_{i j} Q^{i j}
$$

We have for $1 \leqq i \leqq n-1$,

and

$$
\partial_{i} Q=-v_{i n}^{\prime} Q^{i}+\sum_{j \neq i} u_{i j}^{\prime} Q^{i j}=u_{i n} Q^{i}+\sum_{j \neq i} u_{i j}^{\prime} Q^{i j}
$$

$$
\partial_{i}^{2} Q=u_{i n}^{\prime} Q^{i}+\sum_{j \neq i} u_{i j}^{\prime \prime} Q^{i j} .
$$

It follows also from (21) that

$$
\begin{aligned}
& \partial_{n} Q=\sum_{i=1}^{n-1} v_{i n}^{\prime} Q^{i}=-\sum_{i=1}^{n-1} u_{i n} Q^{i} \\
& \partial_{n}^{2} Q=\sum_{i=1}^{n-1} u_{i n}^{\prime} Q^{i}-\sum_{i=1}^{n-1} u_{i n} \partial_{n} Q^{i}=\sum_{i=1}^{n-1} u_{i n}^{\prime} Q^{i}+2 \sum_{i<j}^{n-1} u_{i n} u_{j n} Q^{i j}
\end{aligned}
$$

Now the relation (20) takes the form

$$
\left[\sum_{i=1}^{n-1} \partial_{i}^{2}+\partial_{n}^{2}-2 \sum_{i<j \leqq n-1} u_{i j}, Q\right]=\left(2 \sum_{i=1}^{n-1} u_{i n}\right) Q
$$


Remark that

$$
\left[Q, u_{i j}\right]=u_{i j}^{\prime}\left(Q^{i}-Q^{j}\right)-u_{i j}^{\prime \prime} Q^{i j}
$$

so the left side of $\left(20^{\prime}\right)$ has the form

$$
\begin{aligned}
& \sum_{i=1}^{n-1} 2\left(\partial_{i} Q\right) \partial_{i}+2\left(\partial_{n} Q\right) \partial_{n}+\sum_{i=1}^{n-1} \partial_{i}^{2} Q+\partial_{n}^{2} Q+\sum_{i<j}\left(2 u_{i j}^{\prime}\left(Q^{i}-Q^{j}\right)-2 u_{i j}^{\prime \prime} R^{i j}\right) \\
& =\sum_{i=1}^{n-1}\left(2 u_{i n} Q^{i} \partial_{i n}-2 u_{i n}^{\prime} Q^{i}\right)+\sum_{i<j}^{n-1} 2 u_{i j}^{\prime}\left(Q^{i j} \partial_{i j}+Q^{i}-Q^{j}\right)+2 \sum_{i<j}^{n-1} u_{i n} u_{j n} Q^{i j} .
\end{aligned}
$$

Using the relation $u^{\prime}=-2 v u$ we can rewrite it as

$$
\begin{aligned}
\left(\sum_{i=1}^{n-1} 2 u_{i n}\right) Q & +\sum_{i<j}^{n-1}\left(4 u_{i j}^{\prime}\left(v_{i n}-v_{j n}\right)-2\left(u_{i n}+u_{j n}\right) u_{i j}\right) Q^{i j} \\
& +\sum_{i \neq j \neq k}^{n-1} 2 u_{i j}^{\prime} u_{j k} Q^{i j k}+\sum_{i<j}^{n-1} 2 u_{i n} u_{j n} Q^{i j}
\end{aligned}
$$

It is easy to check by the straightforward calculation that the following identities have a place

$$
\begin{gathered}
\sum_{\sigma \in S_{3}} u_{\sigma(1) \sigma(2)}^{\prime} u_{\sigma(2) \sigma(3)}=0, \\
u_{13} u_{23}+2 u_{12}^{\prime}\left(v_{13}-v_{23}\right)=\left(v_{13}+v_{23}\right) u_{12}
\end{gathered}
$$

for

$$
u=2 \omega^{2} \sinh ^{-2} \omega x, \quad v=2 \omega \operatorname{coth} \omega x .
$$

Thus the previous expression reduces to $\left(\sum_{i=1}^{n-1} 2 u_{i n}\right) Q$. Lemma 1 is proved.

Lemma 2. The operator $D$ defined by (18) satisfies the relation

$$
[\Delta, D]=2 \sum_{i<j}^{n-1} u_{i j} D
$$

Proof. From (20) one can deduce the following chain of relations:

$$
\begin{aligned}
Q_{2}^{1} \circ \Delta & =\left(\Delta-2 u_{12}\right) \circ Q_{2}^{1}, \\
Q_{3}^{12} \circ\left(\Delta-2 u_{12}\right) & =\left(\Delta-2 u_{13}-2 u_{23}\right) \circ Q_{3}^{12}, \quad \text { etc. . }
\end{aligned}
$$

Finally we have

$$
\begin{gathered}
D \circ \Delta=Q_{n}^{1, \ldots, n-1} \circ Q_{n-1}^{1, \ldots, n-2} \circ \cdots \circ Q_{2}^{1} \circ \Delta=\left(\Delta-2 \sum_{i<j}^{n-1} u_{i j}\right) \circ D, \\
\text { i.e. }[\Delta, D]=\sum_{i<j}^{n-1} 2 u_{i j} D .
\end{gathered}
$$

Now let us consider the operator

$$
\sigma_{*}(D)=Q_{\sigma(n)}^{\sigma(1, \ldots, n-1)} \circ Q_{\sigma(n-1)}^{\sigma(1, \ldots, n-2)} \circ \cdots \circ Q_{\sigma(2)}^{\sigma(1)}
$$


for the arbitrary permutation $\sigma \in S_{n}$. It is evident that $\sigma_{*}(D)$ satisfies the same relation (22) as well as the difference $\mathscr{L}=\sigma^{*}(D)-(-1)^{\sigma} D$, which we want to prove to be zero.

Lemma 3. Let $\mathscr{L}$ be a differential operator such that ord $[\Delta, \mathscr{L}] \leqq$ ord $\mathscr{L}$. Then the highest symbol of $\mathscr{L}$ is polynomial on $x$.

Proof. See in [14, Lemma 2.5]. When $x$ tends to infinity staying in the Weyl chamber

$$
C=\left\{x \in \mathbb{R}^{n} \mid x_{1}>x_{2} \cdots>x_{n}\right\}
$$

then $\sigma_{*}(D) \rightarrow(-1)^{\sigma} \prod_{i<j}\left(\partial_{i j}-2 \omega\right)$, as it follows from the definition of $D$. It means that $\mathscr{L} \rightarrow 0$ when $x \rightarrow \infty, x \in C$.

Comparing this fact with Lemma 3 we come to $\mathscr{L}=0$ and formula (19). Theorem 3 is proved.

Example. For $A_{2}$ the formulas (16) lead to the following form of $\psi$ :

$$
\begin{aligned}
\psi= & \left(k_{12} k_{13} k_{23}-2 \omega \operatorname{coth} \omega x_{12} k_{13} k_{23} \cdots\right. \\
& +4 \omega^{2} \operatorname{coth} \omega x_{12} \cot \omega x_{13} k_{23}+\cdots \\
& -8 \omega^{3} \operatorname{coth} \omega x_{12} \cot \omega x_{13} \operatorname{coth} \omega x_{23} \\
& \left.-4 \omega^{3} \sinh ^{-1} \omega x_{12} \sinh ^{-1} \omega x_{13} \sinh ^{-1} \omega x_{23}\right) \\
& \times \exp \left(k_{1} x_{1}+k_{2} x_{2}+k_{3} x_{3}\right) .
\end{aligned}
$$

Here $k_{i j}=k_{i}-k_{j}, x_{i j}=x_{i}-x_{j}$ and we omit the terms which differ only by permutation of indices.

\section{Applications to Harmonic Analysis: Zonal Spherical Function and the Inversion of Abel Transformation for the Symmetric Spaces of Type AII}

Let $G / K$ be a symmetric space (SS), $D_{K}(G)$ be the ring of all differential operators on $G$, which are invariant under $G$-left and $K$-right shifts. The zonal spherical function of $X$ is defined $[23,24]$ as joint eigenfunction $\varphi(g)$ of the operators from $D_{K}(G)$, which is bi-invariant under $K$ and normalized by $\varphi(e)=1$. For the SS of noncompact type, i.e. for connected real semisimple Lie group $G$ with a finite center and its maximal compact subgroup, there exists Harish-Chandra formula, giving all spherical functions as an integral over $K$,

$$
\varphi_{\lambda}(g)=\int_{K} \exp ((i \lambda-\rho) H(g k)) d k .
$$

Here $H(g)$ is determined by the representation $g=k \exp H(g) n$ according to Iwasawa decomposition $G=K A N, \mathscr{A}$ is Lie algebra of $A$,

$$
\lambda \in \mathscr{A}_{\mathbb{C}}^{*}, \quad \rho=\frac{1}{2} \sum_{\alpha \in R_{+}} m_{\alpha} \alpha \in \mathscr{A}^{*},
$$


$R_{+}$is the set of positive roots of the Lie algebra $\mathscr{G}$ of the group $G$ with respect to $\mathscr{A}$, $m_{\alpha}$ are the multiplicities (see [13]).

We want to give the explicit formulas for the zonal spherical functions $\varphi_{\lambda}(g)$ in the case of SS of type AII in Cartan notation, i.e. for $X=S U_{2 n}^{*} / S p_{n}$ (see also [26]).

Let $\delta(x)$ and $q(\mu)$ be the following functions

$$
\begin{aligned}
& \delta(x)=\prod_{i<j} \sinh \left(x_{i}-x_{j}\right), \\
& q(\mu)=\prod_{i<j}\left(\left(\mu_{i}-\mu_{j}\right)^{3}-4\left(\mu_{i}-\mu_{j}\right)\right) .
\end{aligned}
$$

Theorem 4. The zonal spherical function $\varphi_{\lambda}(g)$ for $\mathrm{SS} X=S U_{2 n}^{*} / S p_{n}$ of type AII has the form

$$
\varphi_{\lambda}(g)=\prod_{j=1}^{n}(2 j-1) ! \delta^{-2}(x) q^{-1}(i \lambda) \sum_{\sigma \in S_{n}}(-1)^{\sigma} \psi(i \sigma(\lambda), x),
$$

where $\lambda=\left(\lambda_{1}, \ldots, \lambda_{n}\right) \in \mathbb{C}^{n}, g \in A: g=\exp \operatorname{diag}\left(x_{1}, \ldots, x_{n}, x_{1}, \ldots, x_{n}\right), \sum_{i=1}^{n} x_{i}$ $=0$, and $\psi$ is given by (17).

The proof is a simple consequence of the previous considerations and Gindikin-Karpelevic formula for $c$-function (see [13]). Notice that $K$-biinvariant function on $G$ is completely determined by its restriction to $A=\exp \mathscr{A}$, because $G=K A K$.

For $n=3$ and $n=4$ similar formulas can be extracted from the papers by Vretare [15] and Beerends [16].

Our results allows also to invert the integral Abel transformation

$$
(A f)(g)=\exp (\rho, a) \int_{N} f(g n) d n,
$$

where $g=\exp a \in A, a \in \mathscr{A}, \rho, \mathscr{A}$ and $N$ are the same as above. It transforms the space of $K$-biinvariant functions on $G$ into the space of $W$-invariant functions on $A$, where $W$ is the Weyl group of the root system $R$. For our case, i.e. for SS of type AII, for the Abel transformation (24) Beerends found the explicit integral representation [16]. He found also the formulas for the inversion of this transform for $G=S U_{6}^{*}$ and $S U_{8}^{*}$.

Theorem 5. The following operator

$$
B=c_{n} \delta^{-2}(x) D_{n} \circ \cdots \circ D_{2} \prod_{i<j}\left(\frac{\partial}{\partial x_{i}}-\frac{\partial}{\partial x_{j}}\right),
$$

where $c_{n}^{-1}=2^{n(n-1)} \prod_{j=1}^{n}(2 j-1)$ ! and $D_{i}$ are the same as in $(17)$, is the inversion of Abel transform (24) for $X=S U_{2 n}^{*} / S p_{n}$. More precisely: the function $B(A f)$ coincides with the restriction of K-biinvariant function $f$ on $A=\left\{\exp \operatorname{diag}\left(x_{1}, \ldots, x_{n}\right.\right.$, $\left.\left.x_{1}, \ldots, x_{n}\right), \sum x_{i}=0\right\}$.

The proof follows from Beerends results [16], reducing the problem about the inversion of $A$ to the construction of the so-called shift operator $[19,20]$. In our case it has a form (25). 


\section{References}

1. Dubrovin, B.A., Matveev, V.B., Novikov, S.P.: Nonlinear equation of KdV type, finite-zone linear operators and abelian varieties. Russ. Math. Surv. 31, 51-125 (1976)

2. Chalykh, O.A., Veselov, A.P.: Commutative rings of partial differential operators and Lie algebras. Preprint of FIM (ETH, Zürich), 1988; Commun. Math. Phys. 126, 597-611 (1990)

3. Krichever, I.M.: Methods of algebraic geometry in the theory of nonlinear equations. Russ. Math. Surv. 32 (1977)

4. Feldman, J., Knörrer, H., Trubowitz, E.: There is no two-dimensional analogue of Lamé's equation. Preprint of FIM (ETH, Zürich) 1991; Math. Ann. 294, 295-324 (1992)

5. Sutherland, B.: Exact results for a quantum many-body problem in one-dimension. Phys. Rev. A4, 2019-2021 (1976); Phys. Rev. A5, 1372-1376 (1972)

6. Calogero, F.: Solution of the one-dimensional $n$-body problem with quadratic and/or inversely quadratic pair potentials. J. Math. Phys. 12, 419-436 (1971)

7. Moser, J.: Three integrable Hamiltonian systems, connected with isospectral deformations. Adv. Math. 16, 1-23 (1978)

8. Olshanetsky, M.A., Perelomov, A.M.: Completely integrable Hamiltonian systems associated with semisimple Lie algebra. Invent. Math. 37, 93-108 (1976)

9. Olshanetsky, M.A., Perelomov, A.M.: Quantum completely integrable systems connected with semisimple Lie algebras. Lett. Math. Phys. 2, 7-13 (1977)

10. Olshanetsky, M.A., Perelomov, A.M.: Quantum integrable systems related to Lie algebras. Phys. Rep. 94, 313-404 (1983)

11. Berezin, F.S., Pokhil, G.P., Finkelberg, V.M.: Schrödinger equation for the system of onedimensional particles with point interaction. Vestnik Mosk. Univ. 21-28 (1964) (in Russian)

12. Olshanetsky, M.A., Perelomov, A.M.: Quantum systems related to root systems and radial parts of Laplace operators. Funct. Anal and its Appl. 12, 57-65 (1978)

13. Helgason, S.: Differential geometry, Lie groups and symmetric spaces. New York: Academic Press 1978

14. Berezin, F.A.: Laplace operators on semisimple Lie groups. Proc Mosc. Math. Soc. 6, 371-463 (1957)

15. Vretare, L.: Formulas for elementary spherical functions and generalized Jacobi polynomials. SIAM J. Math. Ann. 15 (4), 805-833 (1984)

16. Beerends, R.: On the Abel transformation and its inversion. Comp. Math. 66, 145-197 (1988)

17. Heckman, G.J., Opdam, E.M.: Root systems and hypergeometric functions I. Comp. Math. 64, 329-352 (1987)

18. Heckman, G.J.: Root system and hypergeometric function II. Comp. Math. 64, 353-373 (1987)

19. Opdam, E.M.: Root systems and hypergeometric functions III, IV. Comp. Math. 67, 21-49, 191-209 (1988)

20. Opdam, E.M.: Some applications of hypergeometric shift operators. Invent. Math. 98, 1-18 (1989)

21. Heckman, G.J.: An elementary approach to the hypergeometric shift operators of Opdam. Invent. Math. 103, 341-350 (1991)

22. Chalykh, O.A.: On one construction of the commutative rings of partial differential operators. (To appear in Math. Notes)

23. Helgason, S.: Groups and geometry analysis. New York: Academic Press 1984

24. Gelfand, I.M.: Spherical functions on the symmetric Riemannian spaces. Dokl. AN SSSR, 70/1, 5-8 (1950)

25. Styrkas, K.: Commutative rings of differential operators, Lie algebras and groups, generated by reflections. Diplom work, Moscow State University 1992 (to be published in Math. Notes)

26. Veselov, A.P., Chalykh, O.A.: Explicit formulas for spherical functions on symmetric spaces of type AII. Funct. Anal. and its Appl. 26/1, 59-61 (1992) 